\title{
A STABLE RECURSIVE ALGORITHM FOR EVALUATION OF ULTIMATE RUIN PROBABILITIES
}

\author{
By Marc Goovaerts and Florian De Vylder* \\ K.U. Leuven and U.C. Louvain, Belgium
}

\begin{abstract}
Probabilities of ruin are solutions of differential or integrodifferential equations. Solving such equations numerically can be performed by means of approximate quadrature formulae for the convolution part of the equation. In this contribution it is shown how applicable recursion formulae, giving results within a prescribed tolerance level, can be obtained. Some numerical results are displayed.
\end{abstract}

\section{KEYWORDS}

Ruin probability, numerical analysis, ordering of risks.

\section{INTRODUCTION}

Gerber (1982) introduced a method for approximating the distribution of aggregate claims and their corresponding stop-loss premium by means of a discrete compound Poisson distribution and its corresponding stop-loss premium. This discretization is an important step in the numerical evaluation of the distribution of aggregate claims, because recent results on recurrence relations for probabilities by PANJER (1981) and SUNDT and JEWELL (1981) only apply to discrete distributions. The discretization technique is efficient in a certain sense, because a properly chosen discretization gives raise to numerical upper and lower bounds on the stop-loss premium, giving the possibility of calculating the numerically estimates for the error on the final numerical results. For calculating the infinite time ruin probability numerically one has to solve the following integral equation, according to GERBER (1979):

$$
\psi(x)=\frac{\lambda}{c} \int_{0}^{x} \psi(x-y)(1-F(y)) d y+\frac{\lambda}{c} \int_{x}^{\infty}(1-F(y)) d y
$$

with

$$
\psi(0)=\frac{\lambda p}{c}, \quad c=\lambda p(1+\eta)
$$

and where

$$
p=\int_{0}^{\infty}(1-F(y)) d y
$$

\footnotetext{
* We would like to thank the referees for some remarks on an earlier draft of the paper.
} 
In the sequel we write $h(x)$ for

$$
h(x)=\int_{x}^{\infty}(1-F(y)) d y
$$

such that $h(0)=p$.

In fact, for solving equation (1), use could be made of a discretization technique for approximating the integrals in the equation in order to get a system of linear equations in the unknown probabilities $\psi\left(x_{1}\right), \psi\left(x_{2}\right), \ldots, \psi\left(x_{n}\right)$. However, not every discretization, is effective to get numerically stable results, on the contrary the propagation of the error induced by the discretization technique provides us, in general, with unstable numerical results. In this contribution we will derive an efficient discretization technique which allows us to calculate $\psi(x)$ numerically within a given tolerance by means of a stable recusive algorithm. As by-product of the method an estimate for the error in the numerical result is obtained.

\section{AN EFFICIENT DISCRETIZATION FOR THE CONVOLUTION INTEGRAL}

In the sequel we define the convolution product by

$$
\varphi * H(x)=\int_{0}^{x} \varphi(x-y) d H(y)
$$

where the integral is taken over the closed interval. The iterative procedure for calculating numerically the infinite time ruin probabilities is based on the following result:

THEOREM. The function $\psi$ (denoting the infinite time ruin probability) is a solution of the equation

$$
\psi *(p-h)=p(1+\eta) \psi-h .
$$

In that equation or in any equivalent equation replace $(h, p)$ by $\left(h_{0}, p_{0}\right)$ and let $\psi_{0}$ be the corresponding solution. Then $\psi \leqslant \psi_{0}\left(\psi \geqslant \psi_{0}\right)$ if $h_{0}$ is decreasing $h_{0} \geqslant 0$, and $h / p \leqslant h_{0} / p_{0}\left(h / p \geqslant h_{0} / p_{0}\right)$.

Proof. See appendix.

COROLLARY. In case $p=p_{0}$ the inequality condition of the theorem reduces to $h \leqslant h_{0}\left(h \geqslant h_{0}\right)$.

REMARKS. For the proof of the theorem to hold it is not necessary that $h_{0}$ can be written as $h_{0}(x)=\int_{x}^{\infty}\left(1-F_{0}(x)\right) d x$ where $F_{0}$ is a distribution function.

In this extended version of the theorem, we do not have the additional assumption $h_{0}(0)=p$. Indeed, $h(0)=p$, so in order to get an upper bound we have to suppose $p \leqslant h_{0}(0)$ and in order to get a lower bound we have to suppose $p \geqslant h_{0}(0)$. 
The theorem, or another version of it, has given us a possibility to obtain analytical upper and lower bounds on infinite time ruin probabilities in case of constraints on claim size distribution, as explained in GoOVAERTS and DE VYLDER (1983). Now it will enable us to deduce numerical bounds on infinite time ruin probabilities because an application of the theorem will provide us with a stable recursive algorithm. Our aim consists in calculating $\psi(x)$. In order to obtain numerical upper and lower bounds (to obtain an error estimate) the following procedure is applied. The underlying motivation of it follows directly from an inspection of figure 1 and an application of the theorem.

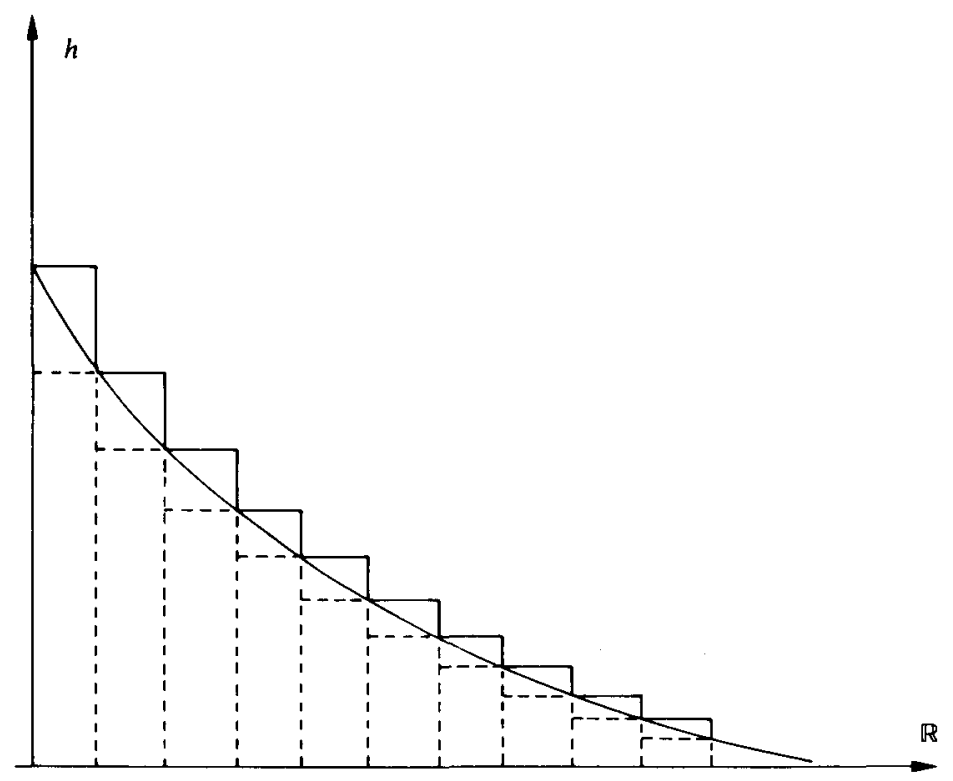

Figure 1.

\section{Practical Procedure}

(i) Consider the following subdivision of the interval $[0, x]$

$$
\left[0, \frac{x}{n}\right],\left[\frac{x}{n}, 2 \frac{x}{n}\right], \ldots,\left[\frac{n-1}{n} x, x\right]
$$

(ii) As indicated in fig. 1 we consider

$$
\begin{gathered}
\forall y \in\left[\frac{i}{n} x, \frac{i+1}{n} x\right] \quad h_{u}(y)=h\left(i \frac{x}{n}\right) \\
h_{l}(y)=h\left((i+1) \frac{x}{n}\right) .
\end{gathered}
$$


Consequently

$$
\begin{array}{ll}
h(y) \leqslant h_{u}(y) & \forall y \geqslant 0 \\
h(y) \geqslant h_{l}(y) & \forall y \geqslant 0 .
\end{array}
$$

(iii) Let $\psi_{u}(x)$ be the solution of

$$
\psi_{u}(k)\left(1-\frac{\lambda^{p} 1}{c}\right)=\frac{\lambda}{c}\left(1-\frac{\lambda^{p} 1}{c}\right) h_{u}(k)-\frac{\lambda}{c} \int_{0}^{k} \psi_{u}^{\prime}(k-y) h_{u}(y) d y .
$$

Because $h_{u}(y)$ is a piecewise constant function, the integral appearing in the r.h.s. can be worked out as follows: let $k=j(x / n)$, then:

$$
\begin{aligned}
\psi_{u}\left(j \frac{x}{n}\right)\left(1-\frac{\lambda^{p} 1}{c}\right)= & \frac{\lambda}{c}\left(1-\frac{\lambda^{p} 1}{c}\right) h\left(j \frac{x}{n}\right) \\
& -\frac{\lambda}{c} \sum_{i=0}^{j-1} h\left(i \frac{x}{n}\right) \int_{i(x / n)}^{(i+1)} \psi_{u}^{\prime}\left(j \frac{x}{n}-y\right) d y .
\end{aligned}
$$

This equation can be cast into the form:

$$
\psi_{u}\left(j \frac{x}{n}\right)=\frac{\lambda}{c} h\left(j \frac{x}{n}\right)+\frac{\lambda}{c} \sum_{i=1}^{j}\left[h\left((i-1) \frac{x}{n}\right)-h\left(i \frac{x}{n}\right)\right] \psi_{u}\left((j-i) \frac{x}{n}\right) .
$$

We also get, proceeding along the same lines,

$$
\begin{aligned}
\psi_{l}\left(j \frac{x}{n}\right)= & \frac{\lambda}{c}\left[1-\frac{\lambda}{c}\left(p_{1}-h\left(\frac{x}{n}\right)\right)\right]^{-1}\left[h\left(j \frac{x}{n}\right)\right. \\
& \left.+\sum_{i=1}^{j-1}\left[h\left(i \frac{x}{n}\right)-h\left((i+1) \frac{x}{n}\right)\right] \psi_{l}\left((j-i) \frac{x}{n}\right)\right]
\end{aligned}
$$

with of course

$$
\psi_{u}(0)=\psi_{l}(0)=\frac{\lambda p}{c}
$$

Starting from (8) we calculate recursively by means of (6) and (7) the couple $\left(\psi_{u}(j(x / n)), \psi_{l}(j(x / n))\right.$ for $j=1,2, \ldots, n$ to obtain two approximations to $\psi(x)$, namely $\psi_{u}^{(n)}(x), \psi_{l}^{(n)}(x)$ where we added explicitly the index $n$ to denote the dependence on $n$. The following inequalities are obtained

$$
\psi_{l}^{(n)}(x) \leqslant \psi(x) \leqslant \psi_{u}^{(n)}(x) .
$$

Also from the result of the above quoted theorem we conclude that

$$
\begin{aligned}
& \psi_{i}^{\left(2^{n}\right)}(x) \text { is not decreasing in } n \\
& \psi_{u}^{\left(2^{n}\right)}(x) \text { is not increasing in } n
\end{aligned}
$$

Hence $\psi(x)$ can be approximated by

$$
\psi(x) \approx \frac{1}{2} \psi_{l}^{(n)}(x)+\frac{1}{2} \psi_{u}^{(n)}(x)
$$


with an upper bound for the error given by

$$
\psi_{u}^{(n)}(x)-\psi_{l}^{(n)}(x)
$$

In order to obtain a result within a prescribed tolerance level $\varepsilon, n$ is chosen large enough such that

$$
\psi_{u}^{(n)}(x)-\psi_{l}^{(n)}(n)<\varepsilon
$$

and $n=2^{k}$ ( $k$ integer).

In order to examine the stability of the numerical procedure, in fact, in order to examine the propagation of errors induced by this recursive algorithm, we suppose that $\psi(x / n), \ldots, \psi((j-1) x / n)$ are calculated with an error $\varepsilon_{1}, \ldots, \varepsilon_{j-1}$. Let $\varepsilon=\max \left\{\varepsilon_{1}, \ldots, \varepsilon_{j-1}\right\}$ then the error $\varepsilon_{j}$ on $\psi(j(x / n))$ is bounded by:

$$
\varepsilon_{j}<\frac{\lambda}{c} \sum_{i=1}^{j}\left(h\left((i-1) \frac{x}{n}\right)-h\left(i \frac{x}{n}\right)\right) \quad \varepsilon_{j}<\varepsilon .
$$

Consequently there is no cumulative effect of propagation of errors. Let us compare the kind of recursion relation with the recursive algorithm of PANJER (1981) and SUNDT and JEWELL (1981) for the calculation of the distribution function of a compound Poisson variable, where

$$
g_{j}=\frac{\lambda}{j} \sum_{i=1}^{j} i f_{i} g_{j-i} .
$$

In case $\lambda$ is relatively small no problem arises as far as the propagation of errors is concerned.

In case $\lambda$ is relatively large however (and this is exactly the case where it is interesting to apply such kind of a scheme) the recursive algorithm is unfortunately not very stable as far as the propagation of errors is concerned. Indeed let $\varepsilon_{1}, \ldots, \varepsilon_{j-1}, \varepsilon_{i}$, denote the errors on $g_{1}, \ldots, g_{j}$ respectively. In case $\varepsilon=$ $\max \left(\varepsilon_{1} \ldots, \varepsilon_{j-1}\right)$ then

$$
\varepsilon_{j} \leqslant \frac{\lambda}{j} \sum_{i=1}^{j} i f_{i} \quad \varepsilon \approx \mu \frac{\lambda}{j} \cdot \varepsilon .
$$

Consequently as long as $j<\lambda$ the upper bound of the error behaves like

$$
\varepsilon_{j} \simeq \lambda^{j} \cdot c
$$

which of course can cause a lot of unexpected difficulties in actual application of the recursion algorithm.

\section{ILLUSTRATION OF THE METHOD}

In VAN Wouwe, De VyLDER and GoovaerTs (1982) the present results are successfully applied to the numerical calculation of bounds on infinite time ruin probabilities in case of constraints on claim size distributions. Use has been made of some of the analytical upper and lower bounds on stop-loss premiums. 
Let us still remark that the rate of convergence of the recursive algorithm is determined by the rate of convergence to zero of the ruin probability when $x \rightarrow \infty$. Therefore we have selected an application which from the numerical point of view has a relatively low speed of convergence.

We consider the case of Pareto claims, $F_{X}(x)=1-1 /(1+x)^{2}, p=1, \eta=0.2$ and of course $h(x)=1 /(1+x)$. The following results are obtained.

\section{Upper Bound}

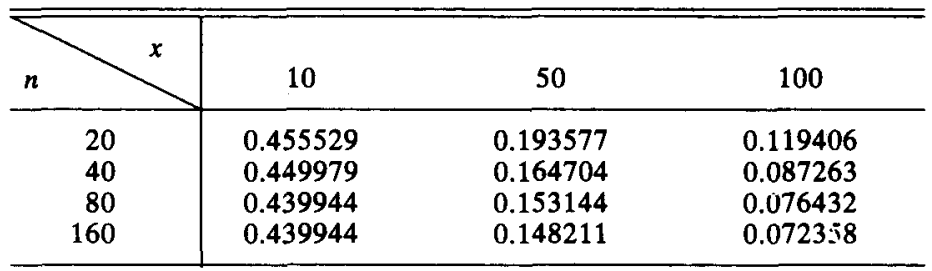

\section{Lower Bound}

\begin{tabular}{r|ccc}
\hline \multicolumn{1}{r|}{} & & & \\
& 10 & 50 & 100 \\
\hline 20 & 0.411083 & 0.121643 & 0.058221 \\
40 & 0.422112 & 0.129821 & 0.061631 \\
80 & 0.428309 & 0.135709 & 0.064429 \\
160 & 0.431619 & 0.139413 & 0.066421 \\
\hline
\end{tabular}

\section{APPENDIX: PROOF OF THE THEOREM}

Use will be made of the following result well known from renewal theory:

LEMMA. If $H$ is a strictly defective distribution function and if $f$ is bounded, then the renewal equation

$$
\xi=f+\xi \times H
$$

has a unique bounded solution $\xi$. If $f \geqslant 0$, then $\xi \geqslant 0$. If $f \leqslant 0$, then $\xi \leqslant 0$.

By means of one partial integration the equation (1) can be cast into the form

$$
\psi *(p-h)=p(1+\eta) \psi-h .
$$

This relation can still be displayed as:

$$
p-p(1+\eta) \psi=(1-\psi) *(p-h) .
$$

By the definition of $\psi_{0}$, we still have

$$
p_{0}-p_{0}(1+\eta) \psi_{0}=\left(1-\psi_{0}\right) *\left(p_{0}-h_{0}\right) .
$$


From (A3) and (A4) we deduce

(A5) $(1+\eta)\left(\psi-\psi_{0}\right)=h / p-h_{0} / p_{0}+\left(\psi-\psi_{0}\right) *(1-h / p)+\psi_{0} *\left(h_{0} / p_{0}-h / p\right)$.

Then the above mentioned lemma can be applied with

$$
\begin{gathered}
\xi=\psi-\psi_{0} \\
H=(1-h / p) /(1+\eta) \\
f=\left(1-\psi_{0}\right) *\left(h / p-h_{0} / p\right) /(1+\eta) .
\end{gathered}
$$

Then of course $f$ is bounded. Moreover because $h / p \geqslant h_{0} / p_{0}$ we have $f \geqslant 0$. The function $H$ is a distribution function (it is increasing and $H \geqslant 0$ ), in fact it is a strictly defective distribution function because $H(\infty)=1 /(1+\eta)$. Hence the result of the theorem follows.

\section{REFERENCES}

Gerber, H. U. (1979) An Introduction to Mathematical Risk Theory. Homewood: Richard D. Irwin, Inc.

Gerber, H. U. (1982) On the Numerical Evaluation of the Distribution of Aggregate Claims and its Stop-Loss Premiums. Insurance: Mathematics and Economics. 1, 13-18.

GoovaerTs, M. and DE VyLDER, F. (1983) Upper and Lower Bounds on Infinite Time Ruin Probabilities in Case of Constraints on Claim Size Distributions. Jounral of Econometrics. 23, $77-90$.

PANJer, H. H. (1981) Recursive Evaluation of a Family of Compound Distributions. Astin Bulletin. $12,22-26$.

SUNDT, B. and JEWELL, W. S. (1981) Further Results on Recursive Evaluation of Compound Distributions. Astin Bulletin. 12, 27-39.

Wouwe, M. VAN, De VyLDER, F. and GoovaerTs, M. (1982) Numerical Bounds on Infinite Time Ruin Probabilities in Case of Constraints on Claim Size Distributions. (To be presented at the 22nd International Congress of Actuaries, Sydney 1984). 